\title{
-NOTES-
}

\section{AN EXAMPLE OF THE NEED FOR TWO STREAM FUNCTIONS IN THREE-DIMENSIONAL FLOWS*}

BY

\author{
Thomas C. Benton (The Pennsylvania State University)
}

The problem of adequate description of the field of flow in a three-dimensional situation where there is no symmetry with respect to an axis cannot be solved with a single stream function. An article by C. S. Yih** gives an account of the theory needed using two stream functions. The author was unable to find any cases where this idea was used, and the following example seems to be about the most simple case in which there is a need for two stream functions and also in which the various differential equations can all be integrated so that a precise picture of the field can be obtained.

Consider a quadruplet source with two perpendicular axes in free space. Take rectangular coordinates $x, y, z$ with the source at the origin. The potential will be given by

$$
\Phi=\frac{\partial}{\partial x}\left(\frac{\partial}{\partial y}\left[r^{-1}\right]\right)
$$

where $r^{2}=x^{2}+y^{2}+z^{2}$, or $\Phi=3 x y / r^{5}$. The velocity components $u, v, w$ are the components of $-\nabla \Phi=3 y\left(5 x^{2}-r^{2}\right) r^{-7} \mathbf{i}+3 x\left(5 y^{2}-r^{2}\right) r^{-7} \mathbf{j}+15 x y z r^{-7} \mathbf{k}$, and the streamlines of the field are solutions of the system:

$$
\frac{d x}{3 y\left(5 x^{2}-r^{2}\right)}=\frac{d y}{3 x\left(5 y^{2}-r^{2}\right)}=\frac{d z}{15 x y z} \text {. }
$$

This system has two integrals. If the three fractions are multiplied respectively by $x, y, z$ and combined in the usual way the result is $r d r / 9 x y r^{2}$; if this is equated to the third fraction the equation $d r / 3 r=d z / 5 z$ results; so $r^{5}=A z^{3}$ is one integral. If the first is multiplied by $x$ and the second by $-y$ and combined the result is $(x d x+y d y) /$ $\left(15 x y\left[x^{2}-y^{2}\right]\right)$; if this is equated to the third fraction $(x d x-y d y) /\left(x^{2}-y^{2}\right)=d z / z$ is obtained; therefore the second integral is $x^{2}-y^{2}=B z^{2}$. So the streamlines are the intersections of the two families;

$$
r^{5}=A z^{3}, \quad x^{2}-y^{2}=B z^{2} .
$$

The first family of surfaces is $\rho^{2}=z^{4 / 5}\left(\alpha^{4 / 5}-z^{4 / 5}\right)$, where $\rho^{2}=x^{2}+y^{2}$ and $\alpha^{2}=A$. For a fixed value of $\alpha$, this surface has intercepts $\rho=0$ at $z=0, \pm \alpha$; and $\rho$ is real only if $-\alpha \leq z \leq \alpha$; and it is a surface of revolution with axis along the axis of $z$. Calculation of the derivative shows the surface is tangent to $z=0$ at the origin; and that $\rho=1 / 5(108 / 5)^{1 / 4} \alpha=0.43 \alpha$, the maximum radius of a circular section perpendicular to the axis of revolution, occurs at $z=(3 / 5)^{5 / 4} \alpha=0.53 \alpha$.

\footnotetext{
${ }^{*}$ Received June 13, 1962; revised manuscript received Oct. 22, 1962. This work was supported by the Ordnance Research Laboratory, Garfield Thomas Water Tunnel, The Pennsylvania State University, under U. S. Navy Contract NOrd 16597.

${ }^{* *}$ Chi-Shun Yih, Stream functions in three dimensional flows, La Houille Blanche, 1957, No. 3, pp. 445-450 (also State University of Iowa, Reprints in Engineering No. 158).
} 
The second family is simply a family of elliptic cones. If $B=\beta^{2}$ the equation becomes $x^{2}=y^{2}+\beta^{2} z^{2}$ so that the plane $x=1$ gives an elliptical section with semi-axes 1 along the $y$-axis and $1 / \beta$ along the $z$-axis. Thus varying $\beta$ only changes the vertical semi-axis of the cone. If $B=-\beta^{2}, y^{2}=x^{2}+\beta^{2} z^{2}$; and then the plane $y=1$ gives the elliptical section.

Suppose that the original form of the equations of the streamlines is used; $A=r^{5} z^{-3}$, $B=\left(x^{2}-y^{2}\right) z^{-2}$, then;

$$
\begin{aligned}
{[\operatorname{grad} A} & =5 r^{3} x z^{-3} \mathbf{i}+5 r^{3} y z^{-3} \mathbf{j}+r^{3}\left(5 z^{2}-3 r^{2}\right) z^{-4} \mathbf{k} \\
\operatorname{grad} B & =2 x z^{-2} \mathbf{i}-2 y z^{-2} \mathbf{j}-2\left(x^{2}-y^{2}\right) z^{-3} \mathbf{k}, \\
\operatorname{grad} A \times \operatorname{grad} B & =4 r^{3} y\left(r^{2}-5 x^{2}\right) z^{-6} \mathbf{i}+4 r^{3} x\left(r^{2}-5 y^{2}\right) z^{-6} \mathbf{j}-20 r^{3} x y z^{-5} \mathbf{k} .
\end{aligned}
$$

But the velocity vector is $-\nabla \Phi=q$ and therefore $\operatorname{grad} A \times \operatorname{grad} B=-(4 / 3) A^{2} q$. If $\lambda=-(3 / 4) A^{-2}$, then $\lambda(\operatorname{grad} A \times \operatorname{grad} B)=q$; but if new functions $\psi, \chi$ are defined so that;

$$
\operatorname{grad} \psi \times \operatorname{grad} \chi=q
$$

it must be true that $\lambda=-(3 / 4) A^{-2}=\psi_{A} \chi_{B}-\psi_{B} \chi_{A}$. If $\chi=B$, then $\chi_{B}=1$ and $\chi_{A}=0$, so $-(3 / 4) A^{-2}=\psi_{A}$ and $\psi=(3 / 4) A^{-1}=(3 / 4) z^{3} z^{-5}$. So the two stream functions are

$$
\psi=(3 / 4) z^{3} r^{-5}, \quad \chi=\left(x^{2}-y^{2}\right) z^{-2} .
$$

Let the three functions $\Phi, \psi, \chi$ be used as curvilinear coordinates in place of $x, y, z$. (This is a departure from Yih's treatment, and although the application to this particular example will be continued, the methods used are quite general). The partial derivatives

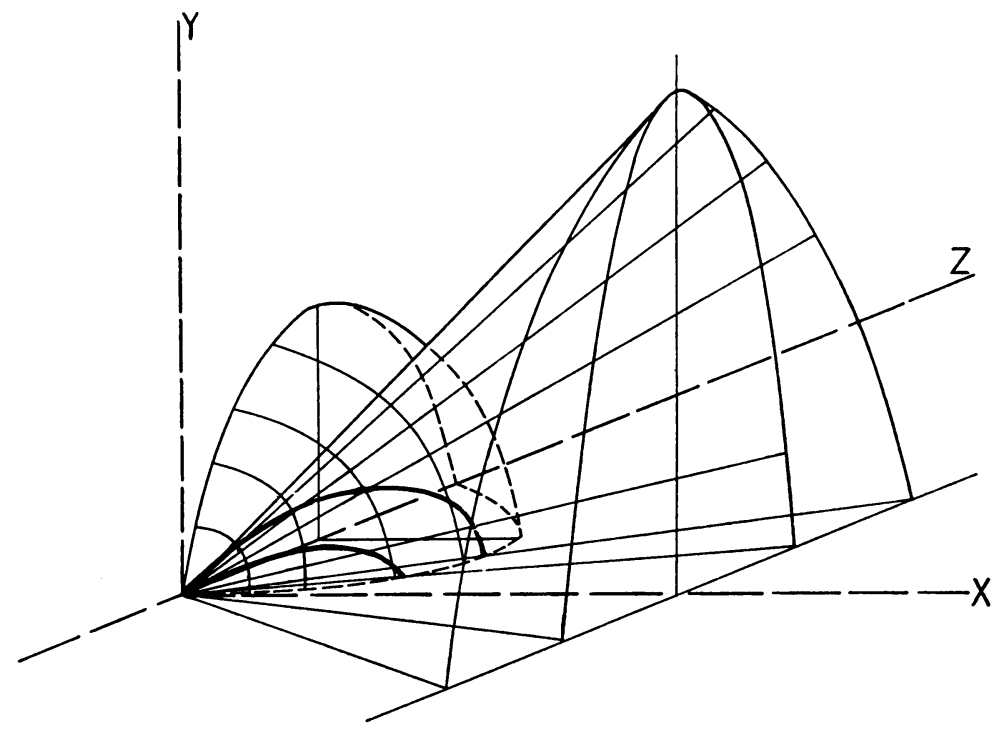

FIg. 1.

of $x, y, z$ with respect to $\Phi, \psi, \chi$ can be calculated from three sets of equations similar to the following set: 


$$
\begin{aligned}
& 0=3 y\left(r^{2}-5 x^{2}\right) r^{-7} \frac{\partial x}{\partial \psi}+3 x\left(r^{2}-5 y^{2}\right) r^{-7} \frac{\partial y}{\partial \psi}-15 x y z r^{-7} \frac{\partial z}{\partial \psi}, \\
& 1=-15 x z^{3} /\left(4 r^{7}\right) \frac{\partial x}{\partial \psi}-15 y z^{3} /\left(4 r^{7}\right) \frac{\partial y}{\partial \psi}+\left(9 z^{2} r^{2}-15 z^{4}\right) /\left(4 r^{7}\right) \frac{\partial z}{\partial \psi}, \\
& 0=2 x z^{-2} \frac{\partial x}{\partial \psi}-2 y z^{-2} \frac{\partial y}{\partial \psi}-2\left(x^{2}-y^{2}\right) z^{-3} \frac{\partial z}{\partial \psi},
\end{aligned}
$$

which are obtained by differentiating $\Phi=3 x y r^{-5}, \psi=(3 / 4) z^{3} r^{-5}, \chi=\left(x^{2}-y^{2}\right) z^{-2}$ with respect to $\psi$. The other sets result from differentiating with respect to $\Phi$ and $\chi$. The Jacobian of $\Phi, \psi, \chi$ with respect to $x, y, z$ has the value:

$$
J=9 r^{-12}\left[5 x^{2} y^{2}-r^{2}\left(x^{2}+y^{2}\right)\right] \text {. }
$$

Now on $\Phi=\Phi_{0}$, the element of length $d s$ is given by $d s^{2}=E d \psi^{2}+2 F d \psi d \chi+G d \chi^{2}$ where $E, F, G$ have the usual meaning in terms of the derivatives with respect to $\psi$, and $\chi$; and the element of surface area is $d \sigma=\left(E G-F^{2}\right)^{1 / 2} d \psi d \chi$. In view of the form of $E, F, G$ this reduces to $J^{-1} \nabla \Phi d \psi d \chi$. The flux through $\Phi=\Phi_{0}$ is $\iint Q \cdot d \sigma=$ $\iint(\nabla \psi \times \nabla \chi) \cdot J^{-1} \nabla \Phi d \psi d \chi$. But $\nabla \Phi \cdot \nabla \psi \times \nabla \chi=J$ so the flux is just $\iint d \psi d \chi$. If the area is bounded by the curves $\psi=\psi_{1}, \psi=\psi_{2}$ and $\chi=\chi_{1}, \chi=\chi_{2}$ the value obtained is $\left(\psi_{2}-\psi_{1}\right) \cdot\left(\chi_{2}-\chi_{1}\right)$, an obvious extension of the property which Stokes' stream function has.

Figure 1 shows two octants of the two stream surfaces with two members of the family of cones but only one of the closed surfaces. The streamlines in which these meet are indicated as heavy curves.

\section{SYMMETRIC DUAL QUADRATIC PROGRAMS*}

\section{By RICHARD W. COTTLE (Operations Research Center, University of California, Berkeley)}

1. Introduction. The duality theory of quadratic programming has been studied by Dennis [1] and principally by Dorn [2, 3, 4]. Wolfe [7] has specialized his results in nonlinear programming to the case of quadratic programming.

In this paper, two quadratic programs are presented which are dual, naturally symmetric, and related to a self-dual quadratic program. It is a consequence of the duality of these programs that if either has an optimal solution, then they share an optimal solution in common. Since duality, symmetry, and self-duality have each been studied by Dorn, some attention is given to the relation between the present work and his.

The programs to be considered are:

Primal Program (P):

$$
\begin{array}{lr}
\text { Minimize } & F(x, y)=\frac{1}{2} y^{\prime} D y+\frac{1}{2} x^{\prime} C x+p^{\prime} x \\
\text { subject to } & D y+A x \geq-b \\
\text { and } & x \geq 0
\end{array}
$$

*Received December 18, 1962; revised manuscript received March 15, 1963. This research has been partially supported by the office of Naval Research under Contract Nonr-222(83) with the University of California. Reproduction in whole or in part is permitted for any purpose of the United States Government. 